\title{
Tratamiento en sesión única de la Aracnofobia en grupo mediante Exposición Rotativa Activa
}

\author{
K. Gunnar Götestam,** \\ Anne Hokstad* \\ * Universidad Noruega de Ciencia y \\ Tecnología (NTNU), Trondheim, \\ NORUEGA \\ ** Universidad de Stanford, California, \\ ESTADOS UNIDOS
}

\begin{abstract}
RESUMEN - La terapia por exposición es el método más eficaz de tratamiento de las fobias específicas y el método de sesión única constituye un avance adicional. Este experimento se concibió en un intento de combinar la observación y el contacto más próximo tanto en la exposición indirecta a través de la observación como en la exposición directa con contacto. Veinticinco pacientes con aracnofobia tomaron parte en el estudio, siendo asignados al azar bien al grupo experimental (a), o bien al grupo de control (b). El diagnóstico se estableció mediante el DSM-III-R y el Cuestionario de Miedos. Los cuestionarios de auto valoración evaluaban la ansiedad cognitiva, los síntomas somáticos, las cogniciones de agorafobia y la auto-eficacia, así como los efectos a largo plazo. Los resultados fueron significativos para todas las variables dependientes. Se obtiene como conclusión que el modelo de "Exposición Rotativa Activa" es un procedimiento rentable en el tratamiento de las fobias específicas.
\end{abstract}

\section{Introducción}

El principio de la exposición es importante en cualquier tipo de terapia de fobias (Marks 1973, 1987). La exposición a la situación que causa el temor, parece ser la terapia más eficaz en las fobias específicas. Las fobias específicas pueden incluso ser tratadas por completo en el transcurso de una sesión única (Öst 1989).
Diversos estudios se han basado en el formato grupal para tratar fobias (Öst 1996). En dos estudios recientes (Öst et al. 1997, Götestam 2001), los pacientes recibían uno de los tres tipos distintos de tratamiento, todos ellos desarrollados durante una sesión de tratamiento de dos horas: exposición en vivo, exposición a una simulación y exposición mediante vídeo. Los tres procedimientos se mostraron eficaces en el tratamiento de la fobia, si bien la exposición en vivo 
pareció ser la más eficaz, mientras que la exposición mediante vídeo se probó como el método menos eficaz.

En un estudio en el que pares de pacientes con fobia fueron tratados en la misma sesión, se evidenció como el efecto de la simulación era muy fuerte, reduciendo la ansiedad en torno al $80 \%$, mientras que la conducta de aproximación y de confrontación con el insecto no variaba mucho mas que en la situación de simulación (Götestam y Berntzen 1987).

El presente experimento se concibió en un intento de combinar la observación y el contacto próximo, tanto en la exposición indirecta a través de la observación como en la exposición directa con contacto. La hipótesis consistía en que una combinación de tratamientos directo e indirecto produciría mejores resultados que el uso únicamente de tratamiento indirecto y que además sería más rentable que si se utilizaba solamente el tratamiento directo.

\section{Material y métodos}

\section{Pacientes}

Veinticinco pacientes con aracnofobia participaron en este estudio sobre tratamiento con una primera parte del estudio que se desarrolló en $1993(n=19)$ y el resto $(n=6)$ transcurridos dos años. Todos los pacientes reunían los criterios del DSM-III-R (APA 1987) acerca de una fobia específica. Algunas de las características demográficas se muestran en la tabla 1 .

\section{Evaluaciones}

DSM-III-R Fobia Específica: En una entrevista clínica abierta se preguntó a los pacientes sobre su fobia, con el fin de determinar si reunían los criterios de diagnóstico de fobia específica (simple).

Cuestionario de Miedos: El grado de ansiedad y de temor de acuerdo con el Cuestionario de Miedos mostró valores de ansiedad de 6,76 y de rechazo de la confrontación de 6,92.

Con anterioridad al tratamiento, así como una semana y un año después de haber sido aplicado, se utilizaron los cuatro cuestionarios siguientes:

Ansiedad Cognitiva: El cuestionario incluía 10 ítems que recogían pensamientos de temor ante la confrontación con el insecto, con respuestas entre 0 (en absoluto) y 4 (en todos los casos) (Öst y Hugdahl 1981). Se utilizó la suma de las puntuaciones de todos los apartados.

Tabla I

Características demográficas

\begin{tabular}{lc} 
Variable & Media/número $(\%)$ \\
\hline Edad & 22,8 años $(17-41)$ \\
Sexo & $24(96 \%)$ \\
$\quad$ Mujeres & $1(4 \%)$ \\
Hombres & $2(8 \%)$ \\
Estado civil & $19(76 \%)$ \\
$\quad$ Casado & $1(4 \%)$ \\
$\quad$ No casado & $(24 \%)$ \\
Viudez & $6(24 \%)$ \\
Situación laboral & $14(56 \%)$ \\
$\quad$ Trabajo & \\
$\quad$ Estudios &
\end{tabular}


Síntomas Somáticos: Otro cuestionario recogía 17 afecciones somáticas relacionadas con la confrontación con el insecto, con respuestas entre 0 (en absoluto) y 4 (mucho) (ibid). Se llevó a cabo la suma de las puntuaciones de todos los apartados.

Auto-Eficacia: Un tercer cuestionario incluía trece pasos de aproximación y la probabilidad (de 0 a 100) de que pudieran llevarse a cabo (Bandura 1986). Se utilizó la suma de la probabilidad de todos los pasos. Únicamente se tuvieron en cuenta los datos previos al tratamiento y los obtenidos transcurrida una semana y ningún dato del segundo cuestionario previo en el grupo de control.

Cuestionario Cognitivo de Agorafobia (ACQ): Se utilizó un cuarto cuestionario con 14 apartados para pensamientos relacionados con la ansiedad fóbica $(1=$ el pensamiento no aparece nunca, hasta $5=$ el pensamiento aparece siempre) (Chambless et al. 1984). De acuerdo con los criterios de Chambless, en esta ocasión consideramos los valores medios de los apartados recogidos.

Test de Conducta de Rechazo (BAT): Se aplicó este test sobre la conducta de rechazo inmediatamente antes y después del tratamiento. Se solicitaba al paciente que se aproximara a la caja abierta tanto como quisiera sin forzarle. La distancia venía a ser de 10 pasos, y 0-10 indicaba el número de pasos de aproximación al insecto que efectuaba el paciente $(0=$ ningún paso, 10 = aproximación hasta el insecto). Adicionalmente, 11 indicaba tocar al insecto, y 12 sostener al insecto sobre o en las manos (Öst 1991). En los casos en que el paciente efectuará más de 9 pasos en el test previo se consideraba que el tratamiento no era necesario.

Evaluación de resultados: Una semana después del tratamiento, los pacientes recibieron un formulario solicitando su valoración de los resultados del tratamiento $(0=$ sin cambios o peor, 1 = alguna mejoría, 2 = mejoría sustancial, 3 = gran mejoría y 4 = "curación total"). Las respuestas a este formulario se enviaban por correo y transcurrido un año se realizaba una evaluación de seguimiento.

Seguimiento: Tras una semana y un año desde el momento del tratamiento se realizaba una evaluación del seguimiento.

\section{Procedimiento}

Procedimiento general: Los pacientes eran registrados de forma consecutiva cuando entraban en contacto con la clínica. Se les remitían los formularios antes indicados y se les pedía que los cumplimentaran y nos los enviaran. Se informaba a los pacientes sobre las bases del tratamiento, es decir, que aproximarse mediante un pequeño paso hacia el insecto causante del temor, con el posterior mantenimiento de dicha aproximación, conduciría finalmente a la disminución de la ansiedad. El insecto causante del temor no se encontraba en la sala de tratamiento inicialmente, y por ello el terapeuta solicitaba la autorización del paciente para introducirlo en la sala un insecto en el interior de una caja de plástico translúcido. En este momento se va acercando a la caja, generándose cierta ansiedad que desaparece al cabo de un rato. La aproximación continuaba, acortando gradualmente la distancia, abriendo la caja, tocando el insecto con un bolígrafo y posteriormente con el dedo. Si era necesario, el terapeuta recreaba los pasos. El objetivo final y más beneficioso para el paciente consistiría en ser capaz de coger al insecto sin llegar a sufrir ansiedad. Esto supone cierto aprendizaje y puede producir buenos resultados tanto a largo como a corto plazo, sin embargo, no siempre es necesario para producir un buen resultado. El modelo para el tratamiento en sesión única fue descrito con anterioridad por Öst (1989). 
El método de "Exposición Rotativa Acti $v a$ ": Se disponía a los pacientes formando un gran círculo alrededor de una mesa pequeña, donde finalmente se coloca la caja con la araña en su interior. Todas las sillas eran azules a excepción de dos rojas. Uno de los pacientes (no el más temeroso) fue seleccionado como ejemplo, sentándose en una de las sillas rojas, siendo sometido a exposición por el terapeuta, que se encontraba sentado en la otra silla del mismo color. En el momento en el que el paciente había tocado la araña por primera vez (o con anterioridad si hacerlo le llevaba más de una hora), se desplazaba una posición al lado contrario del terapeuta y el resto de los pacientes se desplazaban un puesto a la silla siguiente en la misma dirección. A continuación el segundo paciente era sometido a la exposición, hasta que tocaba al insecto (esto solía llevar la mitad del tiempo comparado con el primer paciente). Un segundo desplazamiento de un puesto a la silla siguiente y el tercer paciente era sometido a la exposición real,etc. El cambio de posición se va haciendo cada vez de forma más frecuente y normalmente se completan 2-3 vueltas en el plazo de tres horas. No fue necesario que todos los pacientes tocaran el insecto, ya que a algunos les llevaría demasiado tiempo hacerlo. El cambio de sillas puede realizarse con más celeridad sin necesidad de esperar a que un paciente en concreto tenga el contacto. El efecto de emulación en el grupo resulta determinante y eficaz para facilitar la exposición.

Los grupos tratados estaban formados por 6-8 pacientes. La duración del tratamiento se establecía en tres horas para todos los pacientes, independientemente del resultado del proceso.

Diseño y estadística: Los pacientes fueron asignados al azar a uno de los siguientes grupos: (1) Grupo experimental: Los pacientes $(n=20)$ fueron tratados con exposición inmediatamente después de las evaluaciones previas. (2) Grupo de control: Los pacientes realizaron dos evaluaciones previas en el transcurso de una semana entre ellas, previos a ser tratados mediante exposición.

Se llevó a cabo Análisis de varianzas (ANOVAs) sobre la variable tiempo.

\section{Resultados}

Los resultados para cada una de las variables principales se muestran en la figura 1 .

Ansiedad Cognitiva: Los resultados muestran un descenso claro y significativo de la ansiedad tras el tratamiento $(\mathrm{F}(2,22)=$ $13,43, \mathrm{p}=, 000)$. El grupo de control no varía sustancialmente en el periodo de espera (véase figura 1 a).

Síntomas Somáticos: Los resultados muestran una disminución significativa después del tratamiento $(\mathrm{F}(2,22)=6,191, \mathrm{p}=$ ,007), mientras que el grupo de control también mejora en cierto modo durante el periodo de espera (fig. $1 \mathrm{~b}$ ).

Auto-Eficacia: Los resultados muestran un incremento muy elevado de auto-eficacia tras el tratamiento $(\mathrm{F}(1,13)=90,88, \mathrm{p}=$ ,000). (Esta magnitud no se evaluó por segunda vez antes del tratamiento para el grupo de control) (fig. $1 \mathrm{c}$ ).

Cuestionario de Cogniciones de Agorafo bia $(A C Q)$ : Los valores ACQ van desde 1,71 antes del tratamiento hasta 1,43 después del tratamiento. Esta disminución en los pensamientos relacionados con la fobia fue significativo $(\mathrm{F}(1,14)=7,07, \mathrm{p}=, 019)$.

Test de Conducta de Rechazo (BAT): Existe un elevado incremento en el número de pasos de aproximación efectuados $(\mathrm{F}(1,15)$ $=38,45, \mathrm{p}=, 000)$, mientras que el grupo de 
D

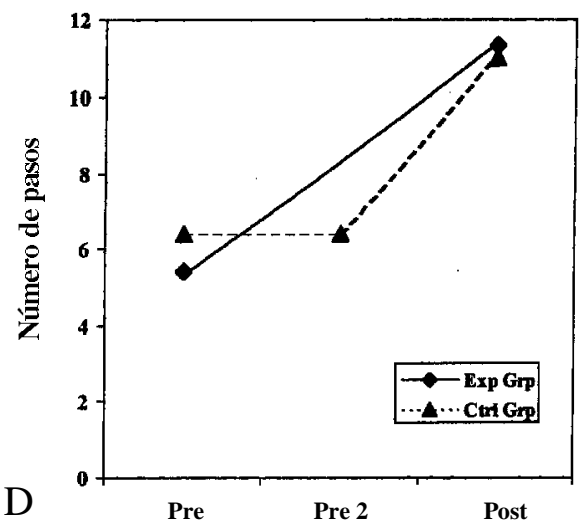

Figura 1. El curso de diferentes variables antes y después de una semana y a los 12 meses en el caso del grupo experimental y con un pre-test adicional para el grupo control. A. Ansiedad cognitiva, B. Síntomas somáticos, C. Auto eficacia, D. Test de evitación conductual.

control no presenta variaciones durante el tiempo de espera (fig. $1 \mathrm{~d}$ ).

Evaluación de resultados: Transcurrida una semana, los resultados $(2,95)$ indican un resultado muy favorable ( $3=$ gran mejoría). Después de un año, este resultado se reduce en cierto grado $(2,60)$, produciéndose un cambio significativo $(\mathrm{F}(1,13)=8,00, \mathrm{p}=$ ,014). Sin embargo, no existe línea de base en esta variable. Con el empleo de una variable de simulación $(0=$ sin cambios como resultado previo), la variable tiempo fue altamente representativa $(\mathrm{F}(12,26)=184,69, \mathrm{p}=, 000)$.

\section{Discusión}

El principal resultado del presente estudio es la presentación método de tratamiento clínicamente útil, la "Exposición Rotativa Activa", que combina la observación con aproximación y confrontación por medio tanto de la exposición indirecta por observación como de la exposición directa por contacto. Esta combinación parece producir mejores resultados que la sola utilización del tratamiento indirecto y además resulta más rentable que el sólo tratamiento directo. 
Con respecto al Cuestionario Cognitivo de Agorafobia (cp Chambless et al. 1984), nuestro grupo de pacientes (que no padecen agorafobia) presentó valores previos un tanto inferiores al grupo de pacientes con agorafobia de Chambless, mientras que los valores que obtuvimos con posterioridad al tratamiento fueron ligeramente superiores a los presentados por el grupo normal de Chambless.

Si comparamos los resultados de este proceso con el grupo de exposición en vivo de Götestam (2001), el presente estudio muestra resultados parecidos pero ligeramente mejores en todos los parámetros, tanto a corto como a largo plazo. Si comparamos los resultados del test de conducta de rechazo (BAT) con los aportados por Öst (1996), ambos se sitúan en el mismo nivel. De esta forma los resultados parecen demostrar la hipótesis de que nos encontramos ante un método de tratamiento eficaz. Calculando los costes, este tratamiento tiene una duración de tres horas y puede ser aplicado hasta a ocho pacientes en una sola sesión, es decir, 22 minutos de tiempo del terapeuta para cada paciente, comparado con el tiempo habitual de una serie compuesta por 6-8 sesiones de una hora de duración.

La conclusión fundamental es que la "Exposición Rotativa Activa" es un método de tratamiento eficaz para las fobias específicas.

\section{Bibliografía}

American Psychiatric Association. Diagnostic and Sta tistical Manual of Mental Disorders. Third, revised edition (DSM-III-R). Washington DC, 1987.

BANDURA, A. Social foundations of thought and action. A social cognitive theory. Englewood Cliffs NJ: Prentice Hall, 1986.
CHAMBleSS, D.L., CAPUTO, G.C., BRIGHT, P., GALLAGHER,R. Assessment of fear in agoraphobics:the Body Sensations Questionnaire and the Agoraphobic Cognitions Questionnaire. Journal of Consulting and Clinical Psychology, 52, 1090-1097, 1984.

GÖTESTAM,K.G. One session group treatment of spider phobia by direct or modeled exposure. Manuscript. 2001.

GÖTESTAM,K.G.,BERNTZEN, D. Use of the modeling effect in one-session exposure. Scandinavian Journal of Behaviour Therapy, 26, 97-101, 1997.

MARKS, I.M. Fears, phobias and rituals. New York: Oxford University Press, 1987.

ÖST, L.G. One-session treatment for specific phobias. Behaviour Research and Therapy, 27, 1-7, 1989.

ÖST, L.G. One-session therapist directed exposure vs. self-exposure in the treatment of spider phobia. Behaviour Research and Therapy, 22, 407-422, 1991.

ÖST, L.G. One-session group treatment for spider phobia. Behaviour Research and Therapy, 34, 707-715 1996 .

ÖST, L.G., HUGDAHL, K. Acquisition of phobias and anxiety response patterns in clinical patients. Behavior Research and Therapy, 19, 439-447, 1981.

ÖST, L.G., FEREBEE, I., FUNNARK, T. One-session group therapy fo spider phobia: Direct versus indirect treatments. Behaviour Research and Therapy, 35,721-732,1997.

Dirección para correspondencia:

K Gunnar Götestam

Department of Psychiatry \& Behavioural Medicine Norwegian University of Science and Technology P O Box 3008 Lade, NO-744 I Trondheim NORUEGA

email: gotestam@medisin.ntnu.no

\section{Nota}

Este proyecto fue patrocinado por la Facultad de Medicina de la Universidad y una versión preliminar del mismo se presentó en el 28 Congreso Europeo de Terapia de Conducta en Corfu, Grecia, en el mes de Septiembre de 1994. 